\title{
LARGE SCALE DISTRIBUTION OF GAS, DUST AND STARS DEDUCED FROM OBSERVATIONS FROM THE UV TO THE IR
}

\author{
J. KOORNNEEF \\ Space Telescope Science Institute \\ 3700 San Martin Drive \\ Baltimore MD 21218 \\ USA
}

\begin{abstract}
We introduce an as yet unpublished set of OAO-II observations of stellar associations in the Large Magellanic Cloud (LMC). Cross-correlation of the photometric characteristics of these fields with the infrared fluxes at these same positions obtained by the IRAS satellite provides information on the local stellar population, the amounts of interstellar extinction and thermal dust emission.
\end{abstract}

\section{IRAS Data}

Infrared data covering the wavelength range from 12 to 100 micron have been obtained with the IRAS satellite. Photometrically significant fluxes in all four channels were observed over an area of approximately 21 square degrees. The useful dynamic range is about a factor of one thousand in each of the channels. The photometric characteristics vary in accord with the wide range of physical conditions sampled. A region measuring 2.5 square degrees around 30 Doradus generates roughly $40 \%$ of the total infared luminosity. With the data smoothed to a resolution of $10 \mathrm{arcmin}$ (equivalent to $154 \mathrm{pc}$ ), the infrared luminosity peaks at $2600 \mathrm{~L}_{\mathrm{Opc}}-2$ at approximately the position of R136 and is also very high for the star-forming region N11 in the far Western side of the Large Cloud. By contrast, the Northern Shapley Constellation III generates only $10 \%$ of the total infrared luminosity from an area of 3.7 square degrees.

\section{OAO-II Observations}

The OAO-II data have been obtained through ten medium bandwith interference filters with centroid wavelengths ranging from 1430 - 4250 . Data are available for a total of 50 fields, each corresponding to a 10-arcmin aperture. Typical observed ultraviolet colours for those fields mimic those of unreddened B3 - B9 stars. About one third of the fields exhibit a significant Balmerdiscontinuity. These fields suffer very little reddening. The ultraviolet fluxes for the remaining fields are dominated by the hot stars typical for young associations. The various colour indices depend strongly on the amount of interstellar extinction. OAO-II magnitudes for this sample range from about 6 to 9 , where the lower limit is set by the instrumental sensitivity. 


\section{Conclusions}

By comparing the IRAS and OAO observations, we find that the respective energy distributions can generally be well understood as resulting from a classic stellar luminosity function reddened by associated dust which, in its turn, radiates in the thermal infrared. Most of the heating photons are provided by $O B$ stars. While the infrared luminosities thus provide a tracer of star formation, our data clearly demonstrate that this relation breaks down in dust-poor regions such as Shapley III. While, for many fields, observed ultraviolet fluxes and colours can be extinction corrected on the basis of dust columns derived from the far IR fluxes, this procedure fails for very high extinction as the ultraviolet fluxes tend to zero. Also, when the stars are located in front of the dust, the correction procedure will lead to an overestimate of the intrinsic ultraviolet fluxes. The ratio of blue over infrared luminosity is therefore rather dependent on the geometry, e.g. the location of OB-associations relative to the parent molecular cloud and, in the case of unresolved galaxies, would change with inclination. 